\title{
La enseñanza de la perspectiva como parte de la fortificación en el siglo XVIII el caso de la Real Academia de Matemáticas de Barcelona ${ }^{1}$
}

\author{
Teachings on perspective as part of \\ fortifications in the 18th century \\ the case of the Royal Academy of Mathematics of Barcelona
}

J ORGE GALINDO DÍAZ

Profesor Asociado - Escuela de Arquitectura y Urbanismo, Universidad Nacional de Colombia

Carrera 27 No. 64 - 60, Manizales, Colombia

jagalindod@unal.edu.co

RESUMO Este artigo pretende demonstrar o modo em que, durante boa parte do século XVIII, o ensino da perspectiva que se levou a cabo nas Academias de engenheiros militares despojando-se do caráter simbólico que teve durante anos para arquitetos, pintores e escultores. Através do estudo do caso da Real Academia de Matemática de Barcelona coloca-se em evidência o modo em que esta disciplina passou a compor, junto com a estática, a mecânica e outras ciências matemáticas, um universo de saberes alcançáveis para os engenheiros da modernidade.

Palavras-chave Perspectiva, Academia de Matemáticas de Barcelona, Tratados de Fortificação

1 Artigo recebido em J ulho de 2008. Autor convidado. 
RESUMEN Este artículo pretende demostrar la manera en que durante buena parte del siglo XVIII, la enseñanza de la perspectiva que se llevó a cabo al interior de las academias de ingenieros militares, tuvo que despojarse del carácter simbólico que tuvo durante años para arquitectos, pintores y escultores. A través del estudio de caso de la Real Academia de Matemáticas de Barcelona se pone en evidencia la manera en que esta disciplina pasó a engrosar, junto con la estática, la mecánica y otras ciencias matemáticas, un universo de saberes alcanzable para los ingenieros de la modernidad.

Palabras clave Perspectiva, Academia de Matemáticas de Barcelona, Tratados de fortificación

ABSTRACT This article seeks to show how during much of 18th century teachings on perspective taking place at the academies of military engineers had to rid itself of the symbolic character it bore during many years for architects, painters, and sculptors. Through the case study of the Royal Academy of Mathematics of Barcelona, it is evident that this discipline became - along with statistics, mechanics, and other mathematical sciences - a reachable universe of knowledge for engineers in modern times.

Keywords Perspective, Academy of Mathematics of Barcelona, Fortification treatises

En los albores del siglo XV se dio inicio en el continente europeo a una transformación tecnológica que habría de afectar las más diversas disciplinas humanas. En 1494 una nueva y poderosa arma batió rápidamente - de manos de las tropas francesas- las hasta ahora muy sólidas murallas italianas. Se trataba del cañón accionado con pólvora. Con él, un novedoso conjunto de conocimientos especializados comenzó a estructurarse. La investigación y la experiencia obtenida en las acciones bélicas permitió conocer los efectos de las nuevas armas, la valoración de los ángulos de tiro y el efecto de las minas; pero fue sin duda en el arte de construcción de fortificaciones en donde se produjo un cambio realmente significativo que tiró por los suelos un conjunto de saberes que hasta ahora se había servido del cuerpo doctrinal de lejanos autores.

No fueron pocos los hombres que a partir de entonces dieron inicio al esfuerzo por lograr un mejor entendimiento de la aplicación de los materiales en la construcción de cortinas y baluartes, del asiento y espesor de cimentaciones y muros, de la profundidad y ancho de los fosos, de la inclinación de taludes, de la resistencia de las bóvedas y forjados, del suministro y evacuación de las aguas, e incluso de la aparentemente mágica 
relación que se establecía entre el trazado a partir de polígonos regulares y el perímetro perfecto capaz de resistir el peor de los asedios. Tal proceso no fue fácil: él demandó una transformación tanto en los métodos de elaboración de ideas y conceptos, como en el manejo de los sistemas de representación gráfica y en la manera de relacionarse con las ciencias. Tuvieron que apropiarse de saberes ajenos e inscribirlos dentro del marco común de las acciones propias del arte de construir y guiarse por la explícita necesidad de definir unos límites propios de su actividad con el fin de conformar un cuerpo doctrinal autónomo capaz de ser transmitido ya no a través del discurso oral (tal y como había sucedido con el conocimiento constructivo medieval) sino mediante la creación de centros de enseñanza. Uno de ellos fue la Real Academia de Matemáticas de Barcelona, creada bajo la sombra de la mentalidad borbónica en 1700 pero que iniciará un fértil proceso de formación de ingenieros militares en 1720 hasta su cierre definitivo en 1803.

\section{Antecedentes: las primeras academias de ingenieros en España}

La referencia más lejana de lo que sería en el siglo XVIII la Real Academia de Matemáticas de Barcelona es la que funcionó intermitentemente en el viejo Alcázar de los Austrias en Madrid entre 1583 y 1697: fundada por Felipe II y promovida por J uan de Herrera con la colaboración del italiano Tiburcio Spanochi, su declarado objetivo era el de comenzar a sustituir por españoles a los militares italianos que habían dominado la construcción de fortificaciones y el planeamiento defensivo durante todo el reinado de Carlos I.

En su primera etapa, los estudios estaban estructurados en dos grandes grupos: (1) conocimientos generales -matemáticas y física- y (2) conocimientos aplicados, de índole militar. De los primeros fueron profesores J ulián Firrufino (que impartía el tratado de la esfera), J uan Cedillo Díaz (traductor de libros sobre el arte de navegar) yJ uan Ang el (encargado de explicar los tratados de Arquímedes). De los conocimientos aplicados se encargaba Pedro Rodríguez (quien desarrollaba el tema de los escuadrones y los principios de aritmética). Lastimosamente de ninguno de ellos han quedado apuntes o notas de clase que permitan tener mayor detalle de sus contenidos con la excepción de los que impartía el profesor Cristóbal de Rojas, llevados a la imprenta en 1598, cuya primera parte estaba dedicada por entero a la Geometría de Euclides, conocimiento que el autor consideraba fundamental para la práctica de los ingenieros militares:

Tres cosas han de concurrir en el soldado, o ingeniero, que quiera tratar la materia de fortificación. La primera, saber mucha parte de Matemáticas: si fuere posible, los seis primeros libros de Euclides, y el undécimo y duodécimo (...) La segunda es, la Aritmética, que sirve para dar cuenta del gasto que para 
hacer la fabrica se ofrecerá antes que se haga (...) La tercera, y mas principal para la fortificación, es saber reconocer bien el puesto donde se ha de hacer la fortaleza, o castillo. ${ }^{2}$

Otros profesores en la academia de Madrid fueron Ginés de Roca y Torras, Bautista Labaña, Ginés de Rocamora, el también tratadista Bernardino de Mendoza ${ }^{3}$ y Pedro Ambrosio de Onderiz, este último traductor al castellano de la obra de Euclides. ${ }^{4}$ Casi con seguridad ejercieron también la cátedra J erónimo María Aflitto y J ulio César Firrufino.

Los períodos de duración de los cursos no eran constantes y los tratados impresos se usaban de manera individual y aislada, más como fuentes de autoridad que como fuentes de conocimiento. Se desconocen los detalles de las asignaturas impartidas y mucho menos los medios empleados en la transmisión didáctica, aunque queda claro que en esta primera experiencia porformar ingenieros españoles es notoria la importancia de Euclides como configurador de la base del pensamiento técnico y del desarrollo ingenieril de comienzos del siglo XVI.

Por otra parte, la academia militar en Bruselas, dirigida por Sebastián Fernández de Medrano constituyó un segundo paso adelante en la formación de los ingenieros de la Corona. Su funcionamiento estuvo comprendido entre 1675 y 1706, cuando la ciudad cayó en poder de los ejércitos de la Gran Alianza. Los estudios en este centro se impartían en un curso general de un año de duración para los oficiales y cadetes, y durante algunos años se incluyó un segundo curso complementario. Para el primero, las materias de estudio eran las siguientes: geometría, fortificación y artillería, geografía y arte de escuadronar. En el segundo curso, de perfeccionamiento, se enseñaba la fortificación y el dibujo, la geometría especulativa y el tratado de la esfera y navegación.

Con seguridad que los textos escritos e impresos por el director de la academia eran fuente de primera mano, por lo que es posible creer que sus contenidos expresan el gran cuerpo teórico que se impartió en este centro durante sus escasos años de funcionamiento. La obra de Fernández de Medrano ha sido calificada de prolífica: ${ }^{5}$ más de seis títulos de su autoría fueron impresos entre 1677 y 1700 en Bruselas y Amberes en lenguas

\footnotetext{
ROJ AS, Cristóbal. Teoría y práctica de fortificación. Madrid: Luis Sánchez, 1598.

MENDOZA, Bernardino. Theorica y practica de guerra. Madrid: viuda de P. Madrigal, 1595.

ONDERIZ, Pedro Ambrosio. La perspectiva especularía de Euclides. Madrid: viuda de Alonso Gómez, 1585.

FERNÁNDEZ DE MEDRANO, Sebastián: (1) Rudimentos Geométricos y Militares. Bruselas: Viuda de Vlugart, 1677.

(2) El Práctico Artillero, el Perfecto Bombardero, y el Arquitecto Perfecto en el Arte Militar. Bruselas: Francisco Foppens, 1680. (3) El Ingeniero. primera parte de la Moderna Arquitectura Militar. Dividido en dos tomos, que contienen cinco libros. Bruselas: casa de Lamberto Marchant, 1687. (4) Breve Descripción del Mundo, o Guia Geographica de Medrano. Lo mas principal en verso. Bruselas: Casa de Lamberto Marchant, 1688. (5) L'ingenieur pratique ou l'Architecture militaire et moderne. Bruselas : casa de Lamberto Marchant, 1696? (6) Los seis primeros libros, onze y doze, de los Elementos Geometricos del Famoso Philosopho Euclides Megarense. Bruselas: casa de Lamberto Marchant, sin fecha, pero el privilegio del Rey es de 1701.
} 
castellana y francesa, destacándose de manera especial El Arquitecto Perfecto en el Arte Militar, ${ }^{6}$ el cual ha sido considerado producto final de varias ediciones corregidas y aumentadas a lo largo de cinco libros. En él, su autor desarrolla una completa teoría del diseño y la construcción de fortificaciones que incluye conocimientos de geometría, trigonometría, cálculo y reglas de proporciones; pero no deja de llamar la atención el hecho de que en las explicaciones relacionadas con los procesos y técnicas de puesta en obra, Fernández de Medrano se apoya en cuidadosas representaciones tridimensionales donde aparecen acciones simultáneas en proceso, que a su vez dan cuenta de secuencias claras y precisas de la construcción de murallas y bastiones (figura 1). Por una parte, tal cosa revela el conocimiento que Fernández de Medrano tenía de la obra de otros autores como Stevin, ${ }^{7}$ Manesson Mallet ${ }^{8}$ y Marolois, ${ }^{9}$ especialmente (de los cuales copia literalmente algunas ilustraciones tal como ocurre en las páginas dedicadas a la arquitectura hidráulica), y por otra, constituye una señal del rol que desde entonces tenía la perspectiva como instrumento de representación de la acción.

A la fecha no se ha examinado con detenimiento el legado de la academia militar de Bruselas, aunque su influencia es explícita en tratados impresos como Escuela de Palas, ${ }^{10}$ el cual se precia de ser un curso matemático dividido en XI tratados, el último de los cuales está consagrado al dibujo. EI capítulo X trata de la perspectiva y el capítulo XI del cálculo de volúmenes necesarios en las obras de fortificación.

Entre los discípulos más destacados de esta efímera academia de Bruselas, está la figura de J orge Próspero de Verboom, español de origen flamenco quien fue llamado en 1709 y designado Ingeniero Real de los Ejércitos, Plazas y Fortificaciones, con el fin de estructurar un cuerpo nacional de ingenieros militares que se aprobaría solo hasta 1711. Entre las tareas de Verboom estaba también la de redactar un proyecto de academia para su formación, desligando sus funciones de las de los artilleros, a más de la que fue sin duda una de sus misiones más importantes: la de dirigir los trabajos de construcción de la Ciudadela de Barcelona, lugar donde habría de morir ocupando el más alto cargo de su jerarquía. Verboom proponía un curso que se dictaba a lo largo de tres años, dividiendo cada uno en dos clases que comprendían los siguientes temas: aritmética, geometría, mecánica y maquinaria, fortificación, construcciones civiles y militares y filosofía

6 FERNÁNDEZ DE MADRANO, Sebastián. El Architecto Perfecto en el Arte Militar, dividido en cinco libros. Amberes: H. \& C. Verdussen, 1708.

7 STEVIN, Simon. La Castramétation. Leyden (2a ed.): chez Matthieu \& Bonaventure Elzevier, 1618.

8 MANESSON MALLET, Alain. Les travaux de Mars ou l'art de la guerre. Paris: Frederic Leonard, 1672.

9 MAROLOIS, Samuel. Opera mathematica, ou Oeuvres mathématiques traictans de géométrie, perspective, architecture et fortification. La Haya: Hagae-Comitis, ex officina Hondii, 1614.

10 ANÓNIMO. Escuela de Palas. Milán: Imprenta Real, 1693. 
natural, entre otras cosas, ${ }^{11}$ germen de la Real Academia de Matemáticas de Barcelona.

\section{La Real Academia de Matemáticas de Barcelona}

La Real Academia de Matemáticas de Barcelona fue fundada en virtud de un real despacho del rey Felipe V, el 22 de enero de 1700. Desde esta fecha y hasta octubre de 1705, cuando tuvo que ser cerrada a raíz de la caída de Barcelona en poder de las tropas aliadas que apoyaban al archiduque Carlos, sus directores fueron Francisco Larrando de Mauleón yJ osé Mendoza y Sandoval. Fue reabierta en 1720 bajo la dirección del ingeniero militar de origen italiano Mateo Calabro (1720-1738), quien será reemplazado primero por el aragonés Pedro de Lucuze (1738-1779) y luego por Miguel Sánchez Taramas (1779-1789) y Félix de Arriete (1789-1793). El ocaso de este centro empezó a hacerse evidente en los últimos años del siglo XVIII, hasta entrar a una crisis que la llevará a su cierre definitivo en 1803. Pero será a lo largo del segundo de sus períodos cuando la Academia consolida su papel de único centro de enseñanza de ingenieros militares en toda España, coincidiendo con una intensa actividad en el terreno de la construcción de fortificaciones y de la producción teórica de sus profesores.

Por lo que respecta a la ubicación de la Academia, esta debió ser instalada temporalmente en algún lugar de Barcelona hasta que fue ubicada definitivamente en unos cuartos del arsenal de la Ciudadela a partir de 1724. En el proyecto de Verboom de 1711 se indicaba que la Academia debía estar constituida, al menos, por dos salas bien organizadas y con suficiente iluminación. Una de ellas estaría destinada a las clases de aritmética y de geometría y tendría una capacidad de 30 o 40 alumnos. La otra, albergaría los cursos de dibujo y fortificación, y dispondría de espacio para la mitad de asistentes.

El primer reglamento propiamente dicho de la Real Academia de Matemáticas de Barcelona data del año 1739 con la aparición de una Ordenanza. ${ }^{12}$ En ella no sólo se designaba la presencia de un director general, dos ayudantes y otro director de dibujo, todos ellos ingenieros, sino que incluso la dotaba de un conjunto importante de instrumentos didácticos y científicos: dos globos, una esfera, un semicírculo, un brújulas, un nivel, etc. Pero lo más significativo era que responsabilizaba al director del contenido de los cursos a impartir, y reglaba su duración y contenido, fijaba en cuarenta el número máximo de alumnos -no mayores de 30 años, ni menores de 15-, establecía los requisitos de buena conducta y nobleza para su admisión y facilitaba el

11 Cf. CAPEL, Horacio. De Palas a Minerva. La formación científica y la estructura institucional de los ingenieros militares del siglo XVIII. Barcelona: Serbal - CSIC, 1988.

12 ANÓNIMO. Ordenanza e instrucción para la enseñanza de las Mathematicas en la Real, y Militar Academia que se ha establecido en Barcelona. Barcelona: Francisco Suria, 1739. 
traslado a Barcelona de los escogidos. Pero además amarraba los contenidos académicos a la tradición impresa en los tratados militares:

Para conseguir la enseñanza según esta idea, deberá el Director General elegir los Tratados mas útiles de las Matemáticas, ordenándolos con sucesivo método, para el pronto aprovechamiento de los Académicos (... $)^{13}$

Todo ello pone en evidencia que lo que se transmitía en la Real Academia de Matemáticas de Barcelona era el cuerpo de conocimientos contenido en los tratados de fortificación, con un sentido más que deliberado, impuesto por la mismísima autoridad real. Es más, aclaraba la necesidad de disponer ordenadamente de sus contenidos, e imponía como norma la transcripción de las clases en notas manuscritas.

La Ordenanza también precisaba sobre el contenido de los cursos, que si bien no era propiamente el que impartirá Lucuze en la Academia durante los años de su dirección, constituyó la norma que lo amparó y que seguramente fue redactada por él mismo, quien ya tenía una idea de lo que pensaba enseñar en 1737. En el primer curso debía hacerse un repaso de la aritmética y de la geometría euclideana, así como de la geometría práctica, las secciones cónicas, la trigonometría y los logaritmos. Como complemento se impartirían clases relativas a la descripción del mundo y principios de mecánica celeste. Terminada esta parte, se hacía un examen que evaluaba el aprovechamiento de los alumnos, dictaminándose los que podían pasar al curso siguiente.

En el segundo curso se explicaba la fortificación moderna -regular e irregular, las normas en el ataque y la defensa de las plazas, el modo de acamparse y atrincherarse, las ventajas y defectos de los terrenos, así como las líneas y ángulos empleados en el trazado de los recintos. En el tercer curso:

(...) se dictará la fuerza que se adquiere por medio de las Máquinas, la gravedad, movimiento, celeridad, y equilibrio de los cuerpos: el arte de mover, levantar, conducir, repartir el agua (...) la proporción, y simetría de las cinco ordenes de Arquitectura: la de las varias partes de un Edificio: la descripción de plantas, y perfiles de ellos, así rectos, como oblicuos: la formación de las bóvedas, y arcos mas comunes: el empuje de ellos contra los pies derechos, o muros, que los sostienen; y la robustez que estos han de tener para resistirle: la calidad de los materiales, y el modo de emplearlos en las construcciones de las obras: la forma de hacer seguros los cimientos sobre distintos terrenos $(. . .)^{14}$

El dibujo sería enseñado en el cuarto curso, tratando de los principios del trazado, manejo de colores y signos convencionales y aplicación de

13 ANÓNIMO. Ordenanza e instrucción para la enseñanza de las Mathematicas en la Real, y Militar Academia que se ha establecido en Barcelona. Barcelona: Francisco Suria, 1739; p.24-25.

14 ANÓNIMO. Ordenanza e instrucción para la enseñanza de las Mathematicas en la Real, y Militar Academia que se ha establecido en Barcelona. Barcelona: Francisco Suria, 1739; p.36-37. 
elementos decorativos; además se impartían principios relativos al presupuesto y gasto de las obras de fortificación.

Se puede afirmar, como conclusión, que la Ordenanza establecía como objetivos claros la transmisión de todas aquellas expresiones propias del conocimiento técnico, a excepción de la formación de habilidades artesanales.

Como se ha señalado, la Ordenanza fijaba en cuatro el número de profesores: el director general, el de dibujo y dos ayudantes, que se repartían de acuerdo a los escalafones de la época; sin embargo, las tareas del director eran suficientes con las exigidas por su carácter burocrático, legando la enseñanza directa sobre los otros tres personajes. La labor de Pedro de Lucuze fue duradera: entre 1738 y 1779, sólo abandonó el cargo temporalmente para dirigir en Madrid una sociedad de matemáticas que no tuvo los frutos esperados dejando por entonces de interino al ingeniero Claudio Martel; otros profesores del período de la dirección de Lucuze serían Carlos Saliquet, Manuel Santisteban, J oan Bautista Escofet, Carlos Cabrer y Suñer (quien posteriormente tendría una destacada actuación en el trazado y dirección de obras en Buenos Aires y Montevideo), Manuel Caballero y Miguel Sánchez Taramas, autor de la traducción del tratado del francés Belidor al castellano. ${ }^{15}$

En diciembre de 1751 apareció una nueva ordenanza reafirmando el contenido de los cursos y acomodándose ya de manera precisa a los contenidos impartidos efectivamente por Lucuze. El contenido de esos cursos es conocido hoy en día a través de los apuntes de clase manuscritos conservados en bibliotecas españolas y americanas. Igualmente se renovaban los instrumentos de que se había dotado al centro y se insistía en la importancia del uso de los tratados de fortificación como fuente de los cursos de la Academia.

Sobre estos últimos, conocemos un listado de los libros que se consideraban necesarios en la Real Academia de Matemáticas de Barcelona en 1751, gracias a la transcripción que hace un autor contemporáneo del legajo 570 del Archivo General de Simancas donde se cuentan, entre otros, a los siguientes autores: ${ }^{16}$ Belidor, Deidier, Puysegur, Vauban, Bullet, Navia Osorio, Bion, Gallon, Gautier, y Le Blond, muchos de los cuales son con seguridad los mismos que se pueden consultar en la Biblioteca Militar del Museo de Montjuic en Barcelona, donde hoy reposan los vestigios bibliográficos de la Academia. De acuerdo con la misma fuente, se poseían en 1790, dentro de los fondos de la Academia, obras de los siguientes autores: Palladio, García de Céspedes, Euler, Zaragoza, Newton, Gracián, González

15 SANCHEZ TARAMAS, Miguel. Tratado de fortificación. Barcelona: Thomas Piferrer, 1769.

16 RIERA, J oan. L Academia de Matemàtiques a la Barcelona II-lustrada (1715-1800). In: Actas del II Congreso Internacional de Historia de la Medicina Catalana. Barcelona, junio de 1975, p.73 - 128. 
de Medina Barba, Enríquez de Villegas, Coehorn, Perrault, Ozanam, Vignola, De La Hire, Tosca, Dechales, P. Cataneo, Manesson Mallet, Escalante, Trincano, Fernández de Medrano, Bernardino de Mendoza, Firrufino, Puga y Rojas, De Ville, Theti, Folch, Lechuga, Sala, Ramelli, Caramuel, Alaba, Errard, Larrando de Mauleon, Bails, Sanz, Busca, Lanteri, Marchi, Lorini, Sardi, Mut y Vegecio.

\section{El Curso matemático de Pedro de Lucuze}

El Curso Matemático de Lucuze, impartido probablemente a partir de 1739, comprendía ocho tratados, dando cumplimiento a la ordenanza de ese año: (I) aritmética, (II) geometría elemental, (III) geometría práctica, (IV) fortificación, (V) artillería, (VI) cosmografía, (VII) estática con un apéndice dedicado a la óptica, y (VIII) arquitectura civil. El orden de estos tratados y sus contenidos es siempre el mismo, tal como se puede verificar a través de la consulta de algunos de sus ejemplares en bibliotecas de Madrid y Barcelona.

El tratado I, De la aritmética, está dividido en seis libros, precedidos de una introducción que defiende la importancia de las matemáticas:

En todos tiempos ha sido la Matemática digna y provechosa aplicación a los mayores ingenios, y en todos los tiempos se han conseguido por ella infinitas utilidades para la vida humana, siendo sobre todo tan preciso su conocimiento al Militar, que sin él apenas pueda dar paso con acierto en su profesión (... $)^{17}$

El tratado II, De la geometría especulativa, escrito por Francisco Sanponts Márquez y Aguilar, ${ }^{18}$ está dividido en seis capítulos y un apéndice, precedidos de un conjunto de definiciones básicas y una introducción. A partir de este segundo tratado se hace evidente la relación que existe entre el curso de Lucuze y el que publicara Belidor en $1725,{ }^{19}$ cuya primera parte trata también de la geometría, se divide en ocho libros e incluye también un tratado anexo dedicado a las secciones cónicas. El tratado III, De la trigonometría y la geometría práctica, está dividido en ocho libros, siguiendo también el orden del tratado de Belidor.

El tratado IV, De la fortificación, se divide a su vez en cuatro libros, precedidos de un conjunto de definiciones, tal como lo hacían los textos impresos consagrados a la fortificación. El libro I trata de la fortificación regular; el libro II lo hace de la fortificación irregular a través de 16 capítulos donde describe los elementos de la fortificación; el libro III explica la fortificación

17 ANÓNIMO. Tratado de fortificación o arquitectura militar. Biblioteca de Catalunya: Mss. No. 3463.

18 ANÓNIMO. Tratado segundo: de la geometría especulativa. Biblioteca de la Universidad de Barcelona: Mss. No. 1688.

19 BELIDOR, Bernard F. Nouveau Cours de Mathematique. Paris: chez Nyon, 1725. 
efectiva sobre el terreno, es decir, la que es irregular, y el libro IV contiene los principios de la fortificación ofensiva y de campaña. En este tratado, Lucuze no apela a la autoridad de Belidor, quien no desarrolla en ninguno de sus libros el tema de fortificación de manera autónoma, apoyándose en el legado del español Vicente Tosca, ${ }^{20}$ quien a su vez se apoya claramente en la obra de Milliet Dechales. ${ }^{21}$

En el tratado V, De la artillería, se habla de la pólvora, de la artillería antigua, de las baterías de los cañones, de las minas y contraminas y de los fuegos artificiales. En el tratado VI, De la cosmografía, se explican la esfera celeste, principios de geografía e hidrografía y algunas cosas "pertenecientes al tiempo". Por su parte, el tratado VII, De la estática. consta de una introducción y tres libros que guardan de nuevo una gran fidelidad al Nouveau Cours de Belidor, tanto en el contenido de las explicaciones como en las ilustraciones que copiaban los alumnos de la Academia. En la introducción se advierte:

La estática es una ciencia físico - matemática, que trata de la proporción del movimiento y peso de los cuerpos graves; de suerte que considera a la cantidad en cuanto es grave y movible, y por esto es parte física, en cuanto se considera la gravedad y el movimiento y es parte matemática porque trata de las proporciones de estos pesos y movimientos, y es importantísima en el conocimiento humano, pues todas las cosas están dispuestas en número, medida y peso, y sobre todo es necesaria en la profesión militar para averiguar las fuerzas de los cuerpos móviles y proporcionar contra ellos los cuerpos que deben resistirles (...) ${ }^{22}$

Como apéndice al tratado VII, De la estática, se introduce otro llamado De la Optica, consagrado por entero a la enseñanza de la esta ciencia y de la perspectiva. ${ }^{23}$ Él consta de dos libros: el primero titulado De los principios generales de la óptica, en el cual se incluyen los siguientes capítulos: definiciones, de la naturaleza y propagación de la luz, del color, y de la visión. El segundo libro trata de la perspectiva y se divide a su vez en: definiciones, de los fundamentos de la perspectiva, y de la práctica de la perspectiva.

¿Cuáles son aquí los referentes que alimentan esta parte del Curso Matemático? De acuerdo con la estructura y contenido de la versión manuscrita, es evidente la influencia de la obra de Samuel Marolois en el desarrollo de los principios generales de la óptica aunque también hay que reconocer que la presencia de libros en la biblioteca de la Academia relacionados con el tema, son un indicio de la variedad de fuentes: Traite de Optique de Y.

20 TOSCA, Vicente. Compendio Matemático. Valencia: casa de J oseph García, 1712.

21 DECHALES, Milliet. L'art de fortifier. París: chez Estienne Michalet, 1684.

22 ANÓ NIMO. Tratado 6, de la estática. Biblioteca de la Escuela Técnica Superior de Arquitectura de Barcelona: Mss. No. R531.2

23 ANÓNIMO. Academia militar. Biblioteca del Museo Militar de Montjuic (Barcelona): Mss. No. A10-E01. 
Newton, traducido al francés por M. Coste; ${ }^{24}$ Traite de optique mechanique de Thomin; ${ }^{25}$ Traite de perspective al usage des Artistes, sin autor; Traite de perspective de J eaurat, ${ }^{26}$ entre otros. Pero si de un análisis de fondo se trata, es inevitable hacer referencia al legado de la obra de G. Desargues -conocida a través de su discípulo A. Bosse ${ }^{27}$ y que también hacía parte de la biblioteca de la Real Academia de Matemáticas de Barcelona-, quien ya se había despreocupado de las connotaciones simbólicas de la geometría convirtiendo a la perspectiva en una ciencia autónoma.

En efecto, la intención fundamental de Desargues fue la de establecer una ciencia geométrica general, capaz de servir como base a las operaciones de diversas técnicas que siempre habían poseído sus teorías específicas: la perspectiva, el corte de piezas de cantería o el diseño de relojes de sol. Sin embargo, artistas y escritores de los siglos XVII y XVIII relegaron al francés hasta 1720 cuando Ozanam ${ }^{28}$ en el prefacio de su libro, negó drásticamente el carácter mágico de la perspectiva enfatizando que este arte constituía únicamente un medio para imitar la naturaleza, representando los objetos visibles tal y como aparecen al ojo humano. Y tal es la concepción de la perspectiva que imperaba durante el siglo XVIII y que influyó sobre los contenidos del Curso Matemático de la Academia de Barcelona: la perspectiva se trata a la manera de un conjunto de reglas extremadamente simples por medio de las cuales, a partir de la planta y los alzados de un cuerpo es posible reconstruir su imagen en tres dimensiones siguiendo un conjunto de instrucciones detalladas.

¿Cuáles eran esas reglas que se enseñaban en la Real Academia de Matemáticas? La primera parte se dedicaba a las definiciones básicas, como en la obra de Samuel Marolois, ${ }^{29}$ a través de las cuales se explicaba la importancia de la perspectiva como un instrumento útil al ingeniero, se mencionaba la catróptica (que considera los rayos que emite un cuerpo con el fin de ser observado), la refracción o dióptrica (su propagación) y la escenografía (sensación tridimensional de los objetos). En los fundamentos de daba repaso a los conocimientos sobre geometría plana para luego entrar a la práctica, donde se daba cuenta de la manera en que el rayo que producía un punto se cortaba con el plano del observador formando así la imagen obtenida. Finalmente, se estudiaban los sistemas de perspectiva usando uno o dos puntos de fuga: los ejemplos referenciados tenían que ver con el dibujo de pavimentos, sólidos platónicos y arcos. Como último tema se

24 NEWTON, Isaac. Traité d'Optique (...) translated by M. Coste. Amsterdam: P. Humbert, 1720.

25 THOMIN, Marc Mitoufled. Traité d'O ptique Mechanique. Paris: J.B. Coignart \& A. Boudet, 1749.

26 JEAURAT, Edme-Sebastien. Traité de perspective a l'usage des artistes. Paris: J ombert, 1750.

27 BOSEE, Abraham. Maniere Universelle de M. Desargues pour pratiquer la Perspective par petit-pied comme le geometral. París: s.i., 1648.

28 OZANAM, J acques. La perspective Théorique et Pratique. Paris: J ombert, 1694.

29 MAROLOIS, Samuel. Opera mathematica, ou Oeuvres mathématiques traictans de géométrie, perspective, architecture et fortification. La Haya: Hagae-Comitis, ex officina Hondii., 1614. 
desarrollaba la manera en que se dibujaban las sombras producidas por la luz de una vela sobre un cuerpo.

¿Cómo se llevaba a cabo la transmisión didáctica? El curso dedicado a la óptica y la perspectiva no se diferenciaba de los otros en cuanto a las condiciones pedagógicas. El profesor de turno (probablemente J uan de Surville, durante muchos años) se valía de la exposición magistral en donde secuencialmente exponía los temas sobre una pizarra, haciendo que cada uno de los discípulos construyese un gráfico hasta conformar una plancha única dividida en 28 o 29 cuadrantes (todos idénticos en los manuscritos consultados) que van de lo más simple a lo más complejo (figura 2).

En las jornadas ajenas a la labor curricular muchos de los aprendices participaban con sus dibujos en la elaboración de los planos y esquemas del fuerte de San Fernando de Figueras, cuya construcción se inició en 1753 y que llegó a constituirse en un auténtico campo de prácticas de los estudiantes de la Academia. De ellos quedan también algunos testimonios, lamentablemente ubicados en manos privadas.

Según los archivos encontrados en el transcurso de la investigación en que se soporta esta exposición, no se emplearon modelos a escala (maquetas) ni lentes, ni vidrios, ni espejos, a diferencia de lo que ocurría con el desarrollo de otros temas, en los que se empleaban termómetros, brújulas, compases, relojes, varas, teodolitos y un sin fin de instrumentos de artillería.

¿Cuál era la utilidad futura? Considerando no accidental que el tema de la perspectiva se incluyera a la manera de un apéndice que precedía al tratado dedicado a la arquitectura civil -en cuyo desarrollo era necesario dibujar con rigor fachadas de edificios, molduras, ornamentos y hasta complejos recintos amurallados- "la perspectiva se hace imprescindible para la arquitectura que deriva de ella su orden y su simetría", en palabras de Niceron. ${ }^{30}$ Pero otros problemas más complejos demandaba la atención de los ingenieros: frente a la necesidad de acertar en el lanzamiento de las balas de cañón, requeridos por la necesidad de trazar adecuadamente sobre el terreno aquello que se representaba en los planos, urgidos por cuantificar las obras de construcción, ¿qué tan útil era el uso de la perspectiva de cara a la realidad? Son evidentes las aplicaciones de la geometría y la aritmética prácticas a la medición, la topografía, la elaboración de presupuestos y otros aspectos de la construcción, sin embargo, al menos para la artillería, de poco siven los dibujos en los que las verdaderas dimensiones no existen y donde las líneas paralelas convergen en un punto situado en el infinito.

Es en ese espacio intermedio entre la teoría y la práctica donde surgen y adquieren importancia, los instrumentos de medición: entendidos como

30 NICERON, J ean François. La perspective curieuse ou magie artificiele de effets merveilleux. Paris: J ean Dubteil, 1638; p.34. 
divinos productos del ingenio, aseguran la transición de lo sensible a lo inteligible. Son frecuentes las alusiones al "báculo de J acob", el "paralelogramun" y el "quadrante", tal y como los describió en su momento Robert Fludd, adaptado al castellano en la obra de Andrés García de Céspedes. ${ }^{31}$

También en el complejo proceso del corte de piedras, tan importante en el arte de la fortificación, la perspectiva mostró sus limitaciones y exigió la presencia de una nueva ciencia: la geometría descriptiva, que de la mano de Gaspar Monge proporcionaría los medios para superar con suficiente éxito, las limitaciones que derivaban de una falta de conocimientos prácticos.

De cualquier manera, el uso de la perspectiva como instrumento técnico nunca alcanzaría el grado de importancia que llegaría a adquirir la geometría plana: ella sirvió no solo para trazar las secciones constructivas de las murallas o los cimientos sino que incluso contribuyó durante muchos años a determinar el espesor de los estribos de los arcos y las bóvedas, las dimensiones de las vigas de madera y el grueso de los muros de contención de los suelos, tal y como lo expondría Bernardo Belidor en su tratado de 1729.32

El tratado VIII, De la arquitectura civil, se conoce a través de una versión fechada en $1778 .{ }^{33}$ En el primer libro (de los dos en que se divide el manuscrito) se estudian los órdenes arquitectónicos, no sin analizar antes las partes principales que componen el orden de arquitectura y los tipos de molduras; dedica también un apartado a los ornatos que se suelen aplicar sobre los edificios y trata ampliamente de las proporciones en los edificios. Entre sus fuentes cita a Scamozzi y Vignola. El segundo libro se divide en tres capítulos: el primero trata "de los empujes de las tierras y el modo de hallar el grueso que se ha de dar a los arcos para que puedan sostenerlas; el segundo, de la delineación de los arcos y las bóvedas"; y el tercero "del modo de calcular los gruesos de los pies derechos para sostener el empuje de arcos y bóvedas".

Para el desarrollo estos temas, el Curso Matemático se sirvió de otro tratado también de Belidor y publicado 1729: ${ }^{34}$ además de seguir de forma idéntica la temática, se valió de los mismos tipos razonamientos para sus explicaciones, como en el caso de la estabilidad de los muros de contención a partir del principio de la palanca demostrado a través de problemas sucesivamente más complejos que una vez resueltos derivaban en escolios; trataba también de los procedimientos gráficos empleados en el trazado de los diferentes tipos de arcos y finalmente explicaba la manera de determinar el espesor de los estribos de las bóvedas.

31 GARCÍA DE CÉSPEDES, Andrés. Libro de instrumentos nuevos de geometría. Madrid: J uan de la Cuesta, 1606.

32 BELIDOR, Bernard F. La Science des Ingénieurs. París: J ombert, 1729.

33 ANÓNIMO. Tratado octavo. De la arquitectura civil. Biblioteca del Colegio de Arquitectos de Madrid: Mss. No. $\mathrm{XIX-82.}$

34 BELIDOR, Bernard F. La Science des Ingénieurs. París: J ombert, 1729. 
Se configura de esta manera todo el conjunto de fuentes directas de las que se valió Pedro de Lucuze para estructurar la enseñanza de la academia de Barcelona. Amplio conocedor de la bibliografía técnica de su tiempo, fue también el autor de tres libros impresos ${ }^{35}$ y generó la edición de un texto complementario a cargo de Ignacio de March. ${ }^{36}$

\section{Otras academias de matemáticas en España durante el siglo XVIII}

En el título III de las Ordenanzas de 1751 se trataba de las "escuelas particulares", situadas en las poblaciones de Orán y Ceuta, posesiones españolas en el norte del Africa:

No siendo dable, que todos los Oficiales, y Cadetes, que tuvieren inclinación a las Mathematicas, puedan pasar para su estudio a la Academia de Barcelona, atento a la falta que harían en sus Cuerpos para el regular servicio, sucediendo esto especialmente en las Plazas de Orán, y Ceuta, por lo numeroso de sus Guarniciones, es mi voluntad continúen en una, y otra las Academias particulares de esta Ciencia, que hay erigidas en ellas, al cargo de un Ingeniero, bajo las reglas siguientes $(\ldots)^{37}$

El perío do de estudios se amplió en estos centros a cuatro años, divididos en dos períodos, pero con los mismos contenidos que los establecidos para la Academia de Barcelona:

En estas se darán los mismos asuntos, que quedan expresados para la Matriz de Barcelona y se observará en lo posible lo prevenido para esta, explicándose en el primero, y segundo año lo correspondiente a la primera, y segunda clase, y en los dos siguientes lo perteneciente a las otras dos, guardando en todo el mismo método $(. . .)^{38}$

Existió también la llamada Academia de Matemáticas de Cartagena de Indias ${ }^{39}$, fundada probablemente en el año de 1730 por iniciativa del ingeniero militar J uan de Herrera y Sotomayor, quien desempeñó el cargo de ingeniero desde 1700, siendo nombrado quince años después jefe y

35 LUCUZE, Pedro. Principios de Fortificación, que contienen las definiciones de los términos principales de las obras de Plaza y de Campaña, con una idea de la conducta regularmente observada en el Ataque y Defensa de las Fortalezas. Barcelona: Thomas Piferrer, 1772. LUCUZE, Pedro. Disertación sobre las Medidas Militares que Contiene la razón de preferir el uso de las Nacionales al de las Forasteras. Barcelona: Francisco Suriá y Burgada, 1773. LUCUZE, Pedro. Advertencias para la medida, y cálculo de los desmontes o excavaciones, en terrenos irregulares, con una regla general para todos ellos. Barcelona: Francisco Suriá, 1766.

$36 \mathrm{MARCH}$, Ignacio. Nociones militares o sumplemento a los principios de fortificación del Exmo. Señor Don Pedro Lucuze. Barcelona: Bernardo Pla, 1781.

37 ANÓ NIMO. Ordenanza e Instrucción de su Magestad para la subsistencia, regimen y enseñanza de la Real Escuela, o Academia Militar de Mathematicas establecida en Barcelona. Barcelona: Francisco Suria, 1751; p.93.

38 ANÓ NIMO. Ordenanza e Instrucción de su Magestad para la subsistencia, regimen y enseñanza de la Real Escuela, - Academia Militar de Mathematicas establecida en Barcelona. Barcelona: Francisco Suria, 1751; p.97.

39 GÓMEZ, Carmen. El sistema defensivo americano. Madrid: Ed. Mapfre, 1992. 
director de las tareas de fortificación de la plaza. La academia de Cartagena de Indias habría iniciado sus tareas el 9 de abril de 1731, y las materias que en ella se impartían fueron las siguientes: (1) geometría práctica con sus aplicaciones y aritmética inferior, (2) trigonometría y sus aplicaciones, (3) uso de instrumentos matemáticos, así de gabinete como de prácticas sobre el terreno, (4) método de levantar planos, (5) principios geográficos y de dibujo, necesarios para formar cartas, planos, perfiles y delineaciones de la arquitectura civil y militar, (6) fortificación moderna, enseñando a conocer todas sus partes, sus usos, máximas y delineaciones, tanto en el papel como en el terreno, (7) lecciones de la polémica ofensiva y defensiva, (8) manejo del cartabón, y (9) principios de maquinaria para elevar cuerpos grandes.

La similitud entonces es considerable con el Curso Matemático de Lucuze que es cronológicamente posterior, lo cual parece apuntar en dos sentidos: que ambos cursos se nutrieron más o menos las mismas las fuentes y que en ambos casos el programa de necesidades planteaba resolver en la formación de los ingenieros aspectos prácticamente idénticos.

\section{Conclusión}

Es la Real Academia de Matemáticas de Barcelona y los contenidos del Curso Matemático que en ella se impartió, el escenario donde confluyeron de manera clara los más diversos tratados de la fortificación impresos en la Europa de los siglos XVI, XVII y XVIII. Se advierte allí la firme intención de formar el cuerpo de ingenieros militares españoles en sintonía con las más recientes escuelas del pensamiento técnico y filosófico.

En cuanto a la enseñanza de la perspectiva que se llevó a cabo en esta institución, ella se aparece despojada del carácter simbólico que la dominó durante años, bajo la forma de reglas más o menos simples pero sobre todo transmisibles. Sin embargo, su aplicación a las demandas utilitarias del ingeniero militar y del arquitecto, especialmente en lo tocante a los trabajos de construcción, fue cuando menos limitada y demandó de variados instrumentos prácticos y de nuevas ciencias capaces de superar sus limitaciones. El uso de la perspectiva se confinó entonces, al menos en los tratados técnicos, a la representación de la acción con fines didácticos y expositivos. 

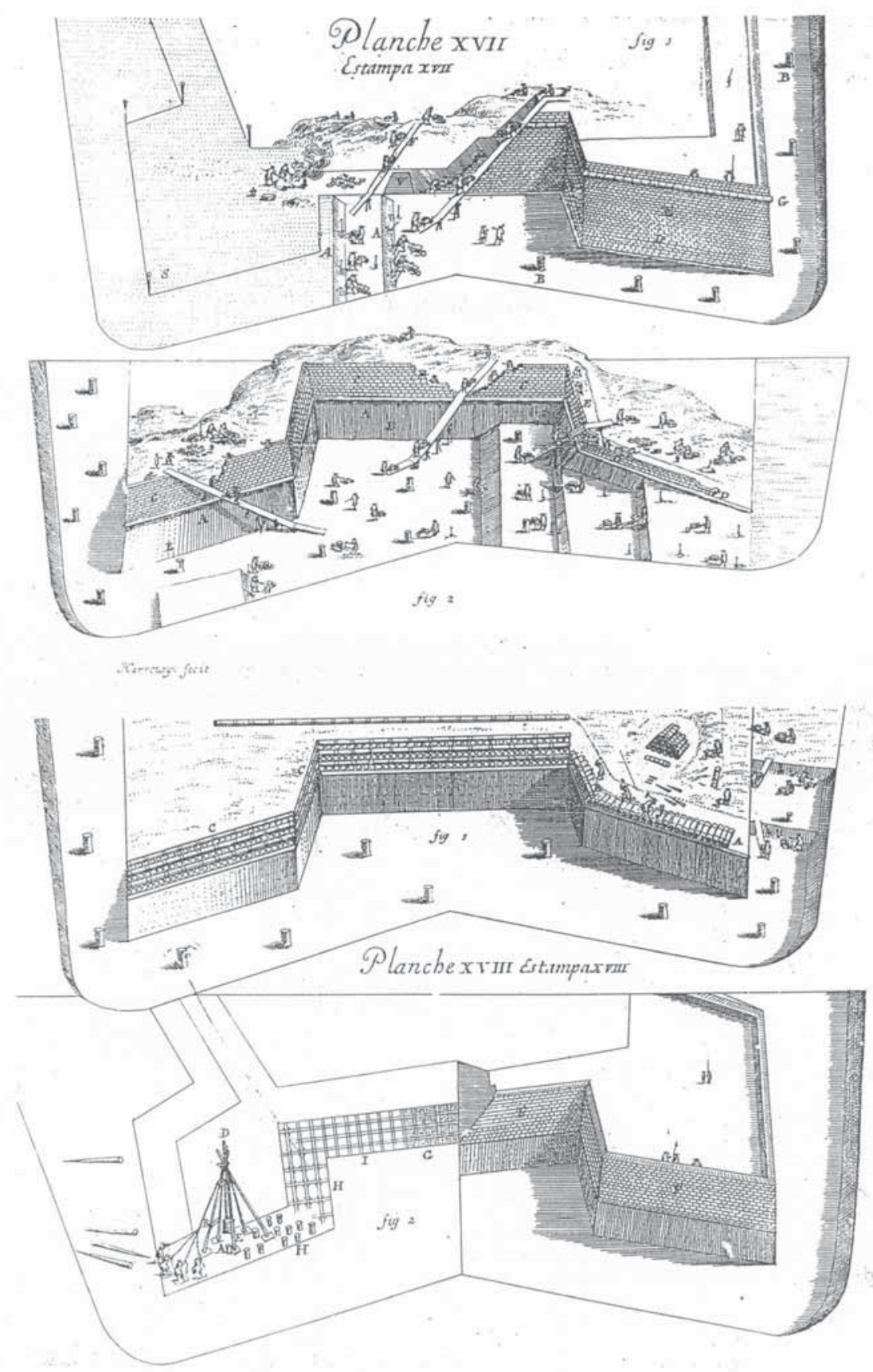

Figura 1: Planchas XVII y XVIII del libro de FERNÁNDEZ DE MEDRANO, Sebastián: EI Arquitec to Perfecto en el Arte Militar. Amberes: H. \& C. Verdussen, 1708. 


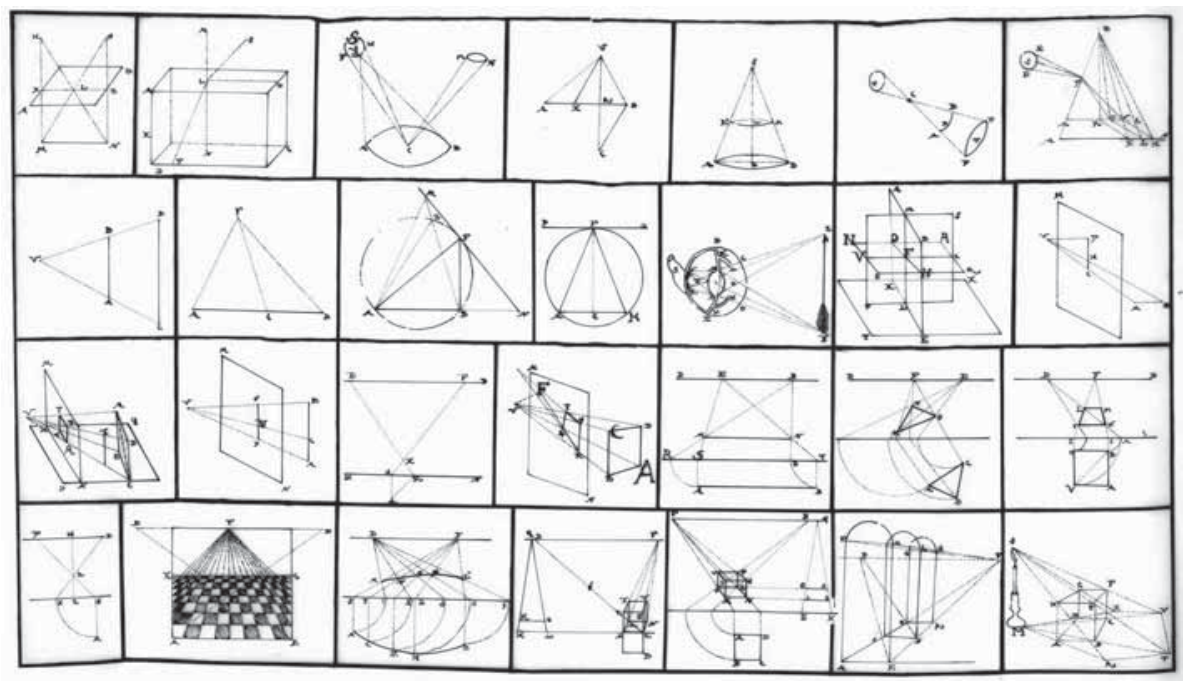

Figura 2: Plancha del tratado De la óptica dictado por Pedro Lucuze en un dibujo manuscrito de un estudiante de la Real Academia de Matemáticas de Barcelona. Biblioteca de la Escuela Técnica Superior de Arquitectura de Barcelona: Mss. No. R531.2, $\sin$ folio, entre folios 32 y 33,28 figuras. 